\title{
Formation and Evolution of Planetary Nebulae: A Radiation Hydrodynamics Study
}

\author{
M. Perinotto \& C. Calonaci \\ Dipartimento di Astronomia, Firenze, Italy \\ D. Schönberner \& M. Steffen \\ Astrophysikalisches Institut Potsdam, Potsdam, Germany \\ T. Blöcker \\ Max-Planck-Institut für Radioastronomie, Bonn, Germany
}

\begin{abstract}
The formation and evolution of a planetary nebula is based on the occurrence of a strong AGB wind and the rapid evolution of the central star with corresponding changes of its ionizing flux and wind power. We have studied the influence of different mass-loss histories in combination with various central-star properties.
\end{abstract}

\section{Model and basic results}

The hydrodynamical equations describing the evolution of a planetary nebula (PN) are solved in spherical geometry by an explicit code based on a secondorder Gudunov-type advection scheme (cf. Perinotto et al. 1998). The radiation part considers time-dependent ionization, recombination, heating and cooling by $\mathrm{H}, \mathrm{He}, \mathrm{C}, \mathrm{N}, \mathrm{O}, \mathrm{Ne}$. The central stars are followed along post-AGB evolutionary tracks of Blöcker (1995). Mass loss rates and velocities of the central-star wind are computed according to the scheme by Marten \& Schönberner (1991), using for the line-driven fast wind the analytical expressions by Kudritzki et al. (1989), with the line-force parameters as recommended by Pauldrach et al. (1988).

We have calculated several hydrodynamical PN sequences corresponding to different AGB wind envelope initial conditions and central star masses. We present here only a single aspect of several results we obtained, namely the evolution of the PN structure for the same AGB wind envelope $\left(\dot{M}=10^{-4} \mathrm{M}_{\odot} / \mathrm{yr}\right.$, $v=15 \mathrm{~km} / \mathrm{s}, \rho \propto r^{-2}$ density law) but different central star masses. In Fig. 1 we compare the resulting PN density and velocity structures at the time when the central star has reached an effective temperature of $50000 \mathrm{~K}$. Only in the $0.605 \mathrm{M}_{\odot}$ case the whole nebula is ionized. In the $0.625 \mathrm{M}_{\odot}$ case the ionization front is just breaking through the outer shock into the still neutral AGB wind. All models, except the one with $0.940 \mathrm{M}_{\odot}$, have already developed a double-shell structure with density maxima defining the inner and outer boundaries of the nebula, the former being caused by the action of the central-star wind which compresses the inner parts of the expanding shell set-up earlier by ionization. Both shells have quite distinct flow properties. 

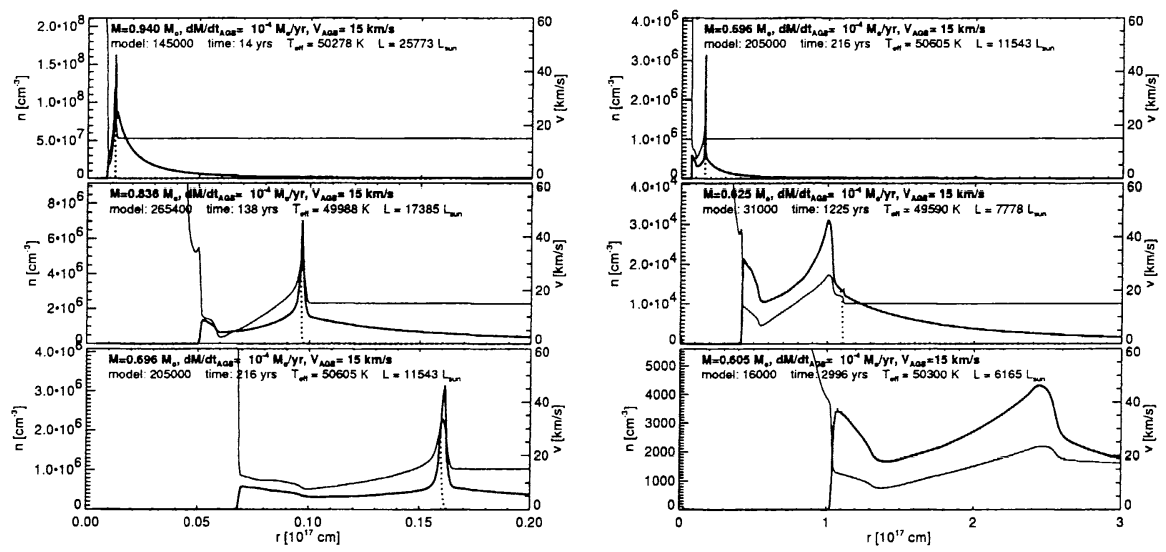

Figure 1. Comparison of nebular structures at $T_{\text {eff }}=50000 \mathrm{~K}$, for different central-stars with the same AGB envelope. Left panel, top to bottom: $M=0.940 \mathrm{M}_{\odot}, L=25800 \mathrm{~L}_{\odot}, t=14$ yrs; $M=0.836 \mathrm{M}_{\odot}$, $L=17400 \mathrm{~L}_{\odot}, t=138 \mathrm{yrs} ; M=0.696 \mathrm{M}_{\odot}, L=11500 \mathrm{~L}_{\odot}, t=$ 216 yrs. Right panel, top to bottom: $M=0.696 \mathrm{M}_{\odot}, L=11500 \mathrm{~L}_{\odot}$, $t=216 \mathrm{yrs} ; M=0.625 \mathrm{M}_{\odot}, L=7800 \mathrm{~L}_{\odot}, t=1225 \mathrm{yrs} ; M=$ $0.605 \mathrm{M}_{\odot}, L=6200 \mathrm{~L}_{\odot}, t=3000$ yrs. Heavy particle densities (thick), electron densities (dotted), and velocities (thin) are shown as a function of radius. Note the different radial coverage of the two sets of figures.

\section{Conclusions}

General conclusions from this study are as follows. The morphologies of the model PNe have no resemblance to previous mass-loss histories; ionization destroys initial density and velocity distribution by setting up a density wave (= shell) traveling outwards; increasing central-star wind power creates a doubleshell structure, which may develop into a thin, single shell if the outer shell is too slow. Simple initial structures may not acquire observed expansion speeds and shell ratios, while models with fast evolving, massive central stars and/or very dense AGB wind envelopes may never become optically thin during the high-luminosity phase of their evolution.

\section{References}

Blöcker, T. 1995, A\&A 297, 727

Kudritzki, R.P., Pauldrach, A., Puls, J., Abbott, D.C. 1989, A\&A 219, 205

Marten, H. \& Schönberner, D. 1991, A\&A 248, 590

Pauldrach, A., Puls, J., Kudritzki, R.P., Mendez, R.M., Heap, S.H. 1988, A\&A 207, 123

Perinotto, M., Kifonidis, K., Schönberner, D., Marten, H. 1998, A\&A 332, 1044 